\title{
Computer Implementation of the Dichotomy Algorithm for Transcendent Equations when Determining the Thread Tension
}

\author{
V. Shcherban, A. Petko, Ju. Makarenko, N. Kolva, D. Egorov \\ Kyiv National University of Technologies and Desing, Ukraine
}

\begin{abstract}
The studies of the computer implementation of the dichotomy algorithm for determining the thread tension on technological equipment determined the values of the thread tension. It is proved that the number of guides on each specific technological machine, the radius of curvature of each guide, the angle of the thread of the guide, the angle of the radial coverage of the thread, the physic-mechanical and structural characteristics of the thread influence the magnitude of the tension of the threads.

The value of the angles of the thread guides and the angles of radial coverage of the thread by the surface of the guide are determined by the geometric parameters and the design of both the filing system on the technological equipment and specific guides. Thanks to this, it became possible at the initial stage of the design of the technological process to determine the tension of the thread in front of the formation zone, depending on the geometric and structural parameters of the equipment and the physico-mechanical and structural characteristics of the thread.

The increase in thread tension occurs due to friction in the contact area with the guides. The magnitude of the friction forces depends on the material of the thread and the guide, the ratio of their geometric dimensions (the radius of the cross section of the thread and the radius of curvature of the guide in the contact zone), the actual angle of the thread of the guide and the angle of the radial coverage of the thread on the surface of the guide, the physicomechanical and structural characteristics of the thread, tension threads in front of the guide. Sequential passage of the thread along the guides, from the entrance zone to the zone of fabric formation and knitwear, leads to a stepwise increase in tension. In this case, the output tension parameter after the previous guide will be an input parameter for the subsequent guide, which allows the use of recursion in determining the tension in front of the formation zone. Keywords: dichotomy algorithm, tension, thread, recursion, guide, radius of curvature, angle of coverage.
\end{abstract}

Statement of the problem, analysis of research and publications. The work [1] is devoted to determining the tension of a thread in interaction with various guides. This study takes into account the real physical and mechanical properties of the threads, namely its bending stiffness, the nonlinear dependence of the friction forces on the normal pressure, the real angle of the thread guide and the angle of the radial surface of the thread guide surface. The effect of kinematic parameters on the value of the thread tension is considered in [2]. In [3], the influence of the thread tension on the amount of deformation of the guide surface is established. Of interest is the work [4], in which the tension is determined depending on the structural characteristics of the thread.

Statement of purpose. The study on the computer implementation of the dichotomy algorithm for determining the thread tension in interaction with the guides of technological equipment should be considered relevant.

The system of equations that describes the change in tension over the zones will have the form 


$$
\begin{aligned}
& P_{1}=P_{0} f_{0}\left(P_{0}\right), \\
& P_{2}=P_{1} f_{1}\left(P_{1}\right) \text {, } \\
& P_{i}=P_{i-1} f_{i-1}\left(P_{i-1}\right), \\
& i=1,2 \ldots n \text {, }
\end{aligned}
$$

where $P_{0}$ - thread tension in the zone of entry into the filing system of the technological machine; $P_{1}, P_{2} \ldots P_{i}$ - thread tension when leaving the corresponding zone; $f_{0}\left(P_{0}\right), f_{1}\left(P_{1}\right) \ldots f_{i-1}\left(P_{i-1}\right)$ functions that bind the thread tension before and after the guide in each zone; $i$ - current zone number; $n$ - the number of guides in the threading system of a particular technological machine.

Using a recursive approach to determining the tension of the thread in front of the fabric and knitwear formation zone, in which the output tension after the guide in the previous zone will be the input tension value in front of the guide in the subsequent zone, we will represent equations (1) in the form

$$
P_{n}=P_{0} \prod_{i=0}^{n} f_{i}\left(P_{i}\right)
$$

The fact is that the dependence that connects the thread tension before and after the guide is a system of two transcendental equations. To determine the root of the transcendental equation in the work, the dichotomy method was used.

Analysis of the conditions of interaction of a thread whose diameter is equal to $2 r$, from $i$ radius guide $R_{i}$ showed that it is necessary to consistently determine its tension in sections $0 \mathrm{~A}$, $\mathrm{AB}$ and B1. It is obvious that at points 0 (input branch that has tension $P_{i-1}$ ) and 1 (output that has tension $P_{i}$ ) the curvature of the monofilament axis is zero. The gravity of the thread can be neglected.

The general system of differential equations in projections on the axis of a natural trihedron, describing the equilibrium of an infinitesimal element of a thread, has the form

$$
\begin{aligned}
& \frac{d P}{d s}-K Q+F_{\tau}=0, \\
& \frac{d Q}{d s}+K P+F_{n}=0, \\
& \frac{d M}{d s}+Q=0, \quad M=B K, B=E I, \\
& K=\frac{1}{\rho}, \rho=\frac{d s}{d \varphi},
\end{aligned}
$$


where $P$ - thread tension;

$s$ - arc coordinate;

$K$ - thread axis curvature;

$Q$ - shear force in the cross section of the thread;

$F_{\tau}, F_{n}$ - projections of external forces on the tangent and normal axis of a natural trihedron;

$M$ - thread bending moment;

$\rho$ - the radius of curvature of the axis of the thread;

$\phi_{i}$ - angle of surface coverage between the initial and final sections of the thread. Considering that external forces do not act on monofilament in sections $0 \mathrm{~A}$ and $\mathrm{B} 1$, we can transform the system of differential equations (3) to the form

$$
\frac{d P}{d s}-K Q=0, \frac{d Q}{d s}+K P=0, \frac{d M}{d s}+Q=0 .
$$

Integration of the system of differential equations (4), taking into account the boundary conditions for points $0, \mathrm{~A}, \mathrm{~B}, 1$, made it possible to obtain the following equations for determining the tension at points $\mathrm{A}$ and $\mathrm{B}$

$$
P_{A}=P_{i-1}-\frac{B}{2\left(R_{i}+r\right)^{2}}, P_{B}=P_{i}-\frac{B}{2\left(R_{i}+r\right)^{2}} .
$$

In turn, the expressions for determining the tension force (always directed tangentially to the axis of the thread) at points A and B can be obtained by parallel transfer of the tension vectors $\vec{P}_{i-1}, \vec{P}_{i}$ at points A and B and decomposing them into tangent and normal using stiffness angles $\gamma_{0 i}, \gamma_{1 i}$. Then we get

$$
P_{A}=P_{0} \cos \left(\gamma_{0 i}\right), P_{B}=P_{1} \cos \left(\gamma_{1 i}\right) .
$$

Solving equations (5) and (6) together, we obtain expressions for determining the stiffness angles

$$
\gamma_{0 i}=\arccos \left[1-\frac{B}{2 P_{i-1}\left(R_{i}+r\right)^{2}}\right], \gamma_{1 i}=\arccos \left[1-\frac{B}{2 P_{i}\left(R_{i}+r\right)^{2}}\right] .
$$

Thus, the actual angle of the thread, taking into account (8), can be determined from the expression

$$
\phi_{i}=\phi_{P i}-\arccos \left[1-\frac{B}{2 P_{i-1}\left(R_{i}+r\right)^{2}}\right]-\arccos \left[1-\frac{B}{2 P_{i}\left(R_{i}+r\right)^{2}}\right],
$$

where $\phi_{P i}$ - estimated value of the angle of coverage $i$ guide.

We proceed to the determination of the tension of the thread in the section $\mathrm{AB}$. In this section, external friction forces will act on the thread $F$ and normal pressure $N$. We assume that the 
friction force is proportional to normal pressure, and the variable is the value of the coefficient of friction, which depends on tension, the radius of curvature of the guide, the angle of the thread of the guide. Based on this, the friction force, taking into account the radial coverage of the thread by the surface of the guide, can be represented as

$$
F=\mu N, \mu=a \frac{4 \sin \left(\frac{\beta_{i}}{2}\right)}{\beta_{i}+\sin \left(\beta_{i}\right)}\left(\frac{P}{R_{i}}\right)^{n_{1}} .
$$

Consider the equilibrium of the element of the thread in section AB. Given that the radius of curvature of the cylinder $R_{i}$ is a transverse force constant $Q=O$. Then, taking into account (10), the system of differential equations (3) takes the form

$$
\begin{aligned}
& \frac{d P}{d s_{i}}-a \frac{4 \sin \left(\frac{\beta_{i}}{2}\right)}{\beta_{i}+\sin \left(\beta_{i}\right)}\left(\frac{P}{R_{i}}\right)^{n_{1}} N=0, \\
& \frac{P}{R_{i}}-N=0, d s_{i}=R_{i} \phi_{i} .
\end{aligned}
$$

We integrate the last differential equation, taking into account the boundary conditions at points $\mathrm{A}$ and $\mathrm{B}$, we obtain

$$
P_{B}^{-n_{1}}-P_{A}^{-n_{1}}=-\frac{4 \sin \left(\frac{\beta_{i}}{2}\right)}{\beta_{i}+\sin \left(\beta_{i}\right)} \frac{n_{1} a \phi_{i}}{R_{i}^{n_{1}}} .
$$

We transform equation (12) using the Lopital rule and find the limit for $n_{1} \rightarrow 0$

$$
P_{B}=P_{A} e^{a \frac{4 \sin \left(\frac{\beta_{i}}{2}\right)}{\beta_{i}+\sin \left(\beta_{i}\right)}\left(\frac{P_{A}}{R_{i}}\right)^{n_{1}} \phi_{i}} .
$$

Solving together (5), (8), (12), we obtain a system of two transcendental equations for the tension of the leading branch of the monofilament $P_{1}$

$$
\begin{aligned}
& P_{i}=P_{i-1} e^{a\left[\frac{P_{i-1}-\frac{B}{2\left(R_{i}+r\right)^{2}}}{R_{i}}\right]^{n_{1}} \phi_{i}}+\frac{B}{2\left(R_{i}+r\right)^{2}}\left\{1-e^{a\left[\frac{P_{i-1}-\frac{B}{2\left(R_{i}+r\right)^{2}}}{R_{i}}\right]^{n_{1}} \phi_{i}}\right\}, \\
& \phi_{i}=\phi_{P i}-\arccos \left[1-\frac{B}{2 P_{i-1}\left(R_{i}+r\right)^{2}}\right]-\arccos \left[1-\frac{B}{2 P_{i}\left(R_{i}+r\right)^{2}}\right] \text {. }
\end{aligned}
$$

Then the connection function of the thread tension before and after the guide will be

$$
f_{i-1}\left(P_{i-1}\right)=e^{a\left[\frac{P_{i-1}-\frac{B}{2\left(R_{i}+r\right)^{2}}}{R_{i}}\right]^{n_{1}} \phi_{i}}+\frac{B}{2 P_{i-1}\left(R_{i}+r\right)^{2}}\left\{1-e^{a\left[\frac{P_{i-1}-\frac{B}{2\left(R_{i}+r\right)^{2}}}{R_{i}}\right]^{n_{1}} \phi_{i}}\right\} .
$$


Figure 1 shows the main forms of the program for determining the tension. Figure 1a shows the results of determining the tension for a nylon complex thread with a design diameter of 0.2 $\mathrm{mm}$. Figure $1 \mathrm{~b}$ shows the results of determining the tension for a nylon monofilament $0.2 \mathrm{~mm}$ in diameter.

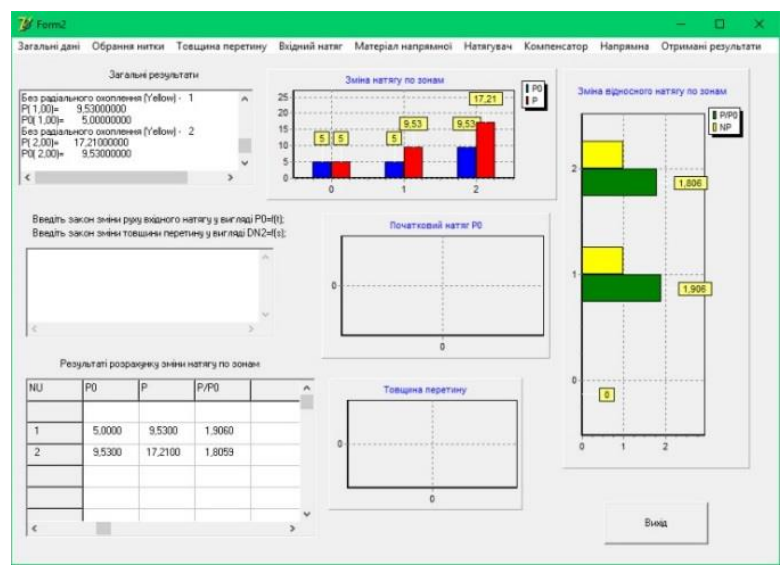

a

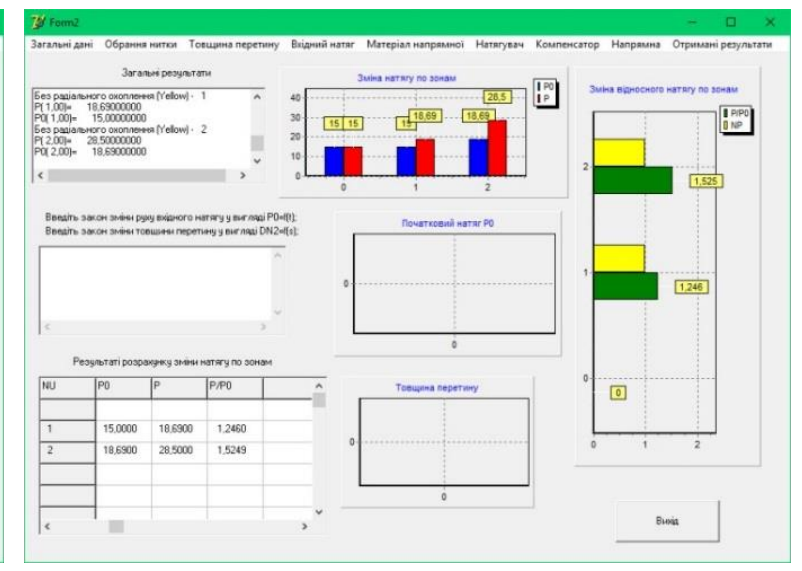

b

Picture 1

Conclusions. Based on the developed block diagram, a computer implementation of the dichotomy algorithm was carried out when determining the tension of the thread in interaction with the guides of the technological equipment. Studies have shown that the number of guides on each specific technological machine affects the tension of the filament in front of the formation zone.

Based on the developed theoretical model, a computational experiment was carried out to determine the tension and the function of linking the tension of the thread before and after the guide, taking into account the radius of curvature of the guide, the angle of the thread of the guide, the angle of the radial coverage of the thread, physical and mechanical and structural characteristics of the thread. It has been established that the value of the angles of filament guides and the angles of radial envelopment of the filament by the surface of the guide are determined by the geometric parameters and design of both the filing system on the technological equipment and specific guides.

\section{References}

1. Scherban V. Interaction yarn guide surface/V.Scerban, M. Sholudko, V. Kalashnik, O. Kolisko//Intellectual Archive, Toronto: Shiny World Corp., Richmond Hill, Ontario, Canada. May 2015. - Volume 4.- Number 3. - P. 10-15.

2. Scherban V. Kinematics of threads cooperates with the guiding surfaces of arbitrary profile /V. Scherban, N. Murza, O. Kolisko, M. Sheludko, I. Semenova //Intellectual Archive, Toronto: Shiny World Corp., Richmond Hill, Ontario, Canada. - May/June - 2016. - Volume 5.- Number 3. - P. 23-27. 
3. Scherban V. Basic parameters of curvature and torsion of the deformable thread in contact with runner/V. Scherban, N.Murza, A.Kirichenko, O. Kolisko, M. Sholudko //Intellectual Archive, Toronto: Shiny World Corp., Richmond Hill, Ontario, Canada. - Nov/Dec - 2016. Volume 5.- Number 6. - P. 18-23.

4. Scherban V. Equalizations of dynamics of filament interactive with surface/V. Scherban, G. Melnik, A.Kirichenko, O. Kolisko, M. Sheludko//Intellectual Archive, Toronto: Shiny World Corp., Richmond Hill, Ontario, Canada. - January/February 2017. - Volume 6.- Number 1. P. 22-26. 